\title{
Comparative Analysis of Big Data
}

\author{
Prayag Tiwari \\ Masters in Innovative Software \\ System (Computer Science Department) \\ National University of Science and \\ Technology MISiS Moscow, Russia,
}

\begin{abstract}
Big Data are turning into another innovation center both in science and in industry and spur innovation movement to information driven design and operational models. We are confronted with a deluge of information produced and caught in computerized structure as a consequence of the headway of sciences, building and innovations, and different social, efficient and human exercises. This big data wonder escorts in another time where human attempts and investigative interests will be helped by not just human capital, and physical and budgetary resources, additionally information resources. The staggering administration produced data turn out to be too huge and complex to be successfully handled by conventional methodologies. The most effective method to store, oversee, and make values from the administration situated big data turn into an imperative exploration issue. In this paper, we addresses a few variables influencing Big Data Quality at different levels, including, gathering, preparing, and capacity. Data quality is critical for all data investigation issues. Generally speaking a Philosophy of Big Data may accommodating in conceptualizing and acknowledging big data science as an administration rehearse, furthermore in transitioning to datarich fates with human and data elements all the more beneficially coinciding in shared development and coordinated effort. We first examine the measurements in big data and big data analysis, and after that emphasis our consideration on the inconsistency problems in big data and the effect of instabilities in big data examination and how to manipulate inconsistencies.
\end{abstract}

\section{Keywords}

Big Data, Data quality, inconsistency in big data, manipulating inconsistencies

\section{INTRODUCTION}

Big Data, likewise alluded to as Data Intensive Technologies, are turning into another innovation pattern in science, industry and business [1,2]. Big Data are getting to be identified with all parts of human movement from simply recording occasions to research, configuration, and generation and advanced administrations or items conveyance to the last shopper. Current advances, for example, Cloud Computing and universal system network give a stage to automation of all procedures in data accumulation, putting away, preparing and representation. One of the most grounded new habitations in contemporary life is big data, vast data sets that might be big in volume, speed, assortment, veracity, and variability. Data volumes and movement are correspondingly "big" in four zones: exploratory, administrative, corporate, and individual data. Gartner's buildup cycle for rising advances examination introduces big data as intersection from the 'top of swelled desire' into the 'trough of bafflement' in 2014 [3]. The targets of big data investigation are changed. They are to a great extent adjusted to the goals of big data partners. These can interpret into making values in medicinal services, quickening the pace of experimental disclosures forever and physical sciences, enhancing the profitability in assembling, building up a focused edge for business, retail, or administration commercial enterprises, and developing in training, media, transportation, or government. The most effective method to better use data resources, notwithstanding physical and monetary resources, and human capital, to make esteem has turned into a fruitful ground for ventures to increase upper hands. As big data examination turns into the following wilderness for headway of learning, advancement, and improved choice making handle, the importance of its effect on the general public as entire can never be disparaged. One ramifications of big data is that people are having a completely distinctive idea and better approach for identifying with data. Where previously everything was signal, now $99 \%$ is clamor, which can prompt overpower, particularly if there is an inability to satisfactorily channel the data.

\section{DEFINITION OF BIG DATA}

Big data is a broad term for data sets so large or complex that traditional data processing applications are inadequate. Challenges include analysis, capture, data curation, search, sharing, storage, transfer, visualization, querying and information privacy [4]. The term regularly alludes essentially to the utilization of prescient examination or certain other progressed strategies to concentrate esteem from information, and sometimes to a specific size of information set. Precision in huge information might prompt more sure choice making, and better choices can bring about more prominent operational proficiency, cost decrease and lessened danger.

\subsection{Characteristics Of Big Data}

Big data characteristics can be described by $5 \mathrm{~V}$

- Volume- The amount of created and stored data. The measure of the data decides the worth and potential understanding and whether it can really be viewed as big data or not.

- Variety- The sort and nature of the data. This people groups who break down it to successfully utilize the subsequent understanding.

- Velocity- The rate at which the data is produced and handled to meet the requests and difficulties that lie in the way of development and advancement.

- Variability- Irregularity of the data routine underpinning shut off processes to handle and manage it. 
- Veracity- The quality of caught data can shift incredibly, influencing exact analysis.

"A new generation of technologies and architectures designed to economically extract value from very large volumes of a wide variety of data by enabling high-velocity capture, discovery, and/or analysis"[5].

It can be supplemented with more basic definition by Jason Bloomberg [6]: "Big Data: a gigantic volume of both organized and unstructured data that is large to the point that it's hard to process utilizing customary database and programming systems." Amid the Big Data lifecycle, every phase of the data change or handling changes the dataset substance, state and hence might change/advance the data model. As a rule there is a need to connection unique data and handled data, keeping referral trustworthiness. This rouses other Big Data highlights: Variability and Linkage or referral respectability. Variability mirrors the way that data are in steady change and might have a distinct state, other than usually characterized as data in move, in rest, or being handled. Supporting these data legitimately will require versatile provenance models and instruments consolidating likewise data respectability and secrecy.

\section{APPLICATIONS DOMAIN AND BEHAVIOR OF BIG DATA}

We watch that Big Data "transformation" is going on in various human movement spaces engaged by critical development of the PC power, universal accessibility of registering and capacity assets, increment of computerized substance generation, versatility. This makes an assortment of the Big Data starting point and utilization spaces. Table 1 records the primary Big Data inception areas and focused on use or application, which are not debilitating and are introduced to represent a requirement for point by point examination of these viewpoints. We allude to the exchange in [7] introduced by the creators at NBDWG about relations between these two measurements to demonstrate their reliance. We can expect high significance of Big Data to business; this really clarifies the current solid enthusiasm to Big Data from business which is really turning into the fundamental main impetus in this innovation space.

Table 1. Big Data Origin And Target Use Domains

\begin{tabular}{|l|l|}
\hline \multicolumn{1}{|c|}{ Big Data Origin } & \multicolumn{1}{c|}{ Big Data Target Use } \\
\hline 1. Science & (a) Scientific discovery \\
2. Telecom & (b) New technologies \\
3. Industry & (c) Manufacturing, process \\
4. Business & control, transport \\
5. Living Environment, & (d) Personal services, campaigns \\
& Cities \\
6. Social media and & (e) Healthcare support \\
networks & \\
7. Healthcare & \\
\hline
\end{tabular}

Science has been generally managing difficulties to handle huge volume of data in complex investigative exploration tests, including likewise wide participation among conveyed gatherings of individual researchers and examination associations. Exploratory research commonly incorporates gathering of data in uninvolved perception or dynamic investigations which plan to check some experimental theory. Logical exploration and disclosure strategies are ordinarily in view of the starting theory and a model which can be refined taking into account the gathered data. The refined model might prompt another more progressed and exact examination and/or the past data re-assessment. The future Scientific Data and Big Data Infrastructure (SDI/BDI) necessities to backing all data taking care of operations and procedures giving additionally access to data and to offices to working together analysts. Other than conventional access control and data security issues, security administrations need to guarantee. In business, privately owned businesses won't regularly share data or skill. At the point when managing data, organizations will plan dependably to keep control over their data resources. They might utilize shared outsider offices, as mists or masters instruments, yet uncommon measures should be taken to guarantee workspace security and data insurance, including info/yield data sterilization. Big Data in industry are identified with controlling complex innovative procedures and items or offices. Advanced PC helped fabricating produces colossal measure of data which are when all is said in done should be put away or held to permit powerful quality control or diagnostics if there should be an occurrence of disappointment or accident. Additionally to e-Science, in numerous mechanical applications/situations there is a requirement for coordinated effort or cooperation of numerous laborers and technologists.

\section{ANALYSIS OF BIG DATA}

Big Data analysis is the procedure of gathering, sorting out and breaking down expansive arrangements of information (called Big Data) to find designs and other valuable data. Big Data analysis can offer associations to better comprehend the data some assistance with containing inside of the information and will likewise recognize the information that is most vital to the business and future business choices. Experts working with enormous information fundamentally need the learning that originates from investigating the information. The analysis of Big Data includes numerous unmistakable stages as appeared in the figure underneath, each of which presents challenges. Numerous individuals tragically concentrate just on the analysis/demonstrating stage: while that stage is significant, it is of little use without alternate periods of the data analysis pipeline. Indeed, even in the analysis stage, which has gotten much consideration, there are ineffectively comprehended complexities in the setting of multi-tenanted bunches where a few clients' projects run simultaneously. Numerous noteworthy difficulties augment past the analysis stage. For instance, Big Data must be overseen in setting, which might be loud, heterogeneous and exclude a forthright model. Doing as such raises the need to track provenance and to handle vulnerability and blunder: points that are vital to achievement, but then once in a while said at the same time as Big Data. Correspondingly, the inquiries to the data analysis pipeline will regularly not all be laid out ahead of time. We might need to make sense of good inquiries in view of the data. Doing this will require more quick witted frameworks furthermore better backing for client connection with the analysis pipeline. Truth be told, we at present have a noteworthy bottleneck in the quantity of individuals engaged to make inquiries of the data and dissect it. We can radically expand this number by supporting numerous levels of engagement with the data, not all requiring profound database aptitude. Answers for issues, for example, this won't originate from incremental enhancements to nothing new, for example, industry might make all alone. Maybe, they oblige us to on a very basic level reexamine how we oversee data analysis. 


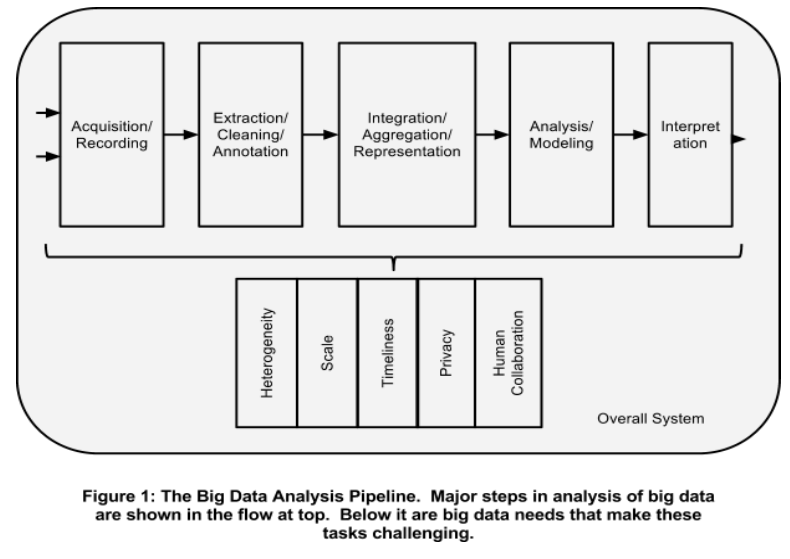

Existing computational procedures can be connected, either as is or with a few expansions, to at any rate a few parts of the Big Data issue. For instance, social databases depend on the idea of sensible data freedom: clients can consider what they need to process, while the framework (with gifted specialists planning those frameworks) decides how to figure it proficiently. So also, the SQL standard and the social data model give a uniform, intense dialect to express numerous question needs and, on a basic level, permits clients to pick between merchants, expanding rivalry. The test in front of us is to consolidate these sound components of earlier frameworks as we devise novel answers for the numerous new difficulties of Big Data. We start by considering the five stages in the pipeline, then proceed onward to the five crosscutting difficulties, and end with a dialog of the engineering of the general framework that consolidates every one of these capacities.

\section{ACQUISITION AND RECORDING OF DATA}

Big Data does not emerge out of a vacuum: it is measured from some data creating source. For instance, consider our capacity to sense and watch our general surroundings, from the heart rate of an elderly subject, and vicinity of poisons noticeable all around we inhale, to the arranged square kilometer exhibit telescope, which will deliver up to 1 million terabytes of crude data every day. Additionally, investigative analyses and reenactments can without much of a stretch produce petabytes of data today. A lot of this data is of no hobby, and it can be sifted and packed by requests of extent. One test is to characterize these channels so as to not dispose of helpful data. For instance, assume one sensor perusing contrasts generously from the rest: it is liable to be because of the sensor being broken, yet in what manner would we be able to make certain that it is not an antiquity that merits consideration? What's more, the data gathered by these sensors regularly are spatially and transiently related (e.g., movement sensors on the same street portion). We require research in the investigation of data decrease that can shrewdly prepare this crude data to a size that its clients can deal with while not missing the needle in the bundle. Moreover, we require "on-line" analysis procedures that can process such gushing data on the fly, since we can't bear to store first and diminish a short time later. The second big test is to consequently produce the right metadata to depict what data is recorded and how it is recorded and measured. For instance, in logical analyses, significant insight in regards to particular trial conditions and systems might be required to have the capacity to translate the outcomes effectively, and it is critical that such metadata recorded with observational data. Metadata securing frameworks can minimize the human weight in recording metadata. Another vital issue here is data provenance. Recording data about the data at its introduction to the world is not helpful unless this data can be deciphered and brought along through the data analysis pipeline. For instance, a preparing mistake at one stage can render consequent analysis futile; with suitable provenance, we can undoubtedly recognize all ensuing handling that reliant on this stride. In this manner we require research both into producing suitable metadata and into data frameworks that convey the provenance of data and its metadata through data analysis pipelines.

\section{EXTRACTION AND CLEANING OF DATA}

Every now and again, the data gathered won't be in an organization prepared for analysis. For instance, consider the accumulation of electronic wellbeing records in a healing center, containing translated transcriptions from a few doctors, organized data from sensors and estimations (conceivably with some related vulnerability), and picture data, for example, x-beams. We can't leave the data in this structure and still adequately investigate it. Maybe we require a data extraction handle that hauls out the required data from the basic sources and communicates it in an organized structure suitable for analysis. Doing this effectively and totally is a proceeding with specialized test. Note that this data additionally incorporates pictures and will later on incorporate video; such extraction is regularly exceedingly application subordinate (e.g., what you need to haul out of a MRI is altogether different from what you would haul out of a photo of the stars, or an observation photograph). What's more, because of the pervasiveness of reconnaissance cameras and notoriety of GPS-empowered cell telephones, cameras, and other convenient gadgets, rich and high loyalty area and direction (i.e., development in space) data can likewise be removed.

We are accustomed to considering Big Data as continually letting us know reality, yet this is really a long way from reality. For instance, patients might stow away hazardous conduct and guardians might now and again mis-analyze a condition; patients might likewise erroneously review the name of a medication or even that they ever took it, prompting missing data in (the history segment of) their medicinal record. Existing work on data cleaning accept very much perceived limitations on legitimate data or surely knew mistake models; for some rising Big Data spaces these don't exist.

\subsection{Integration, Aggregation and Representation of Data}

Given the heterogeneity of the surge of data, it is insufficient only to record it and toss it into a store. Consider, for instance, data from a scope of investigative tests. On the off chance that we simply have a cluster of data sets in a store, it is impossible anybody will ever have the capacity to discover, not to mention reuse, any of this data. With satisfactory metadata, there is some trust, however even along these lines, difficulties will stay because of contrasts in test points of interest and in data record structure.

Data analysis is impressively more difficult than essentially finding, recognizing, understanding, and referring to data. For successful extensive scale analysis the majority of this needs to happen in a totally robotized way. This requires contrasts in data structure and semantics to be communicated in structures that are PC justifiable, and afterward 
"mechanically" resolvable. There is a solid assortment of work in data combination that can give a portion of the answers. In any case, extensive extra work is required to accomplish robotized blunder free distinction determination.

Notwithstanding for less difficult investigations that rely on upon one and only data set, there remains an essential inquiry of suitable database outline. More often than not, there will be numerous option routes in which to store the same data. Certain outlines will have points of interest over others for specific purposes, and potentially disadvantages for different purposes. Witness, for case, the gigantic assortment in the structure of bioinformatics databases with data in regards to considerably comparable elements, for example, qualities. Database configuration is today a craftsmanship, and is precisely executed in the endeavor setting by generously compensated experts. We should empower different experts, for example, area researchers, to make viable database plans, either through concocting apparatuses to help them in the configuration process or through swearing off the outline prepare totally and creating methods so that databases can be utilized successfully as a part of the nonattendance of shrewd database plan.

\subsection{Analysis and Modelling of Data}

Techniques for questioning and mining Big Data are generally not quite the same as customary measurable analysis on little specimens. Big Data is frequently uproarious, rapid, heterogeneous, between related and conniving. By the by, even boisterous Big Data could be more important than modest examples on the grounds that general insights got from incessant examples and connection analysis typically overwhelm singular changes and frequently reveal more solid concealed examples and information. Further, interconnected Big Data frames substantial heterogeneous data systems, with which data repetition can be investigated to adjust for missing data, to crosscheck clashing cases, to approve reliable connections, to unveil inborn bunches, and to reveal shrouded connections and models.

Mining requires incorporated, cleaned, dependable, and productively open data, decisive inquiry and mining interfaces, adaptable mining calculations, and big-data figuring situations. In the meantime, data mining itself can likewise be utilized to enhance the quality and dependability of the data, comprehend its semantics, and give smart questioning capacities. As noted beforehand, genuine therapeutic records have blunders, are heterogeneous, and habitually are conveyed over numerous frameworks. The estimation of Big Data analysis in social insurance, to take only one case application area, must be acknowledged in the event that it can be connected vigorously under these troublesome conditions. On the other side, learning created from data can help in revising blunders and uprooting ambiguity. For instance, a doctor might compose "DVT" as the conclusion for a patient. This shortening is ordinarily utilized for both "profound vein thrombosis" and "diverticulitis," two altogether different restorative conditions. An information base built from related data can utilize related side effects or solutions to figure out which of two the doctor implied.

Big Data is likewise empowering the up and coming era of intelligent data analysis with ongoing answers. Later on, questions towards Big Data will be naturally produced for substance creation on sites, to populate hot-records or suggestions, and to give analysis of the estimation of a data set to choose whether to store or to dispose of it. Scaling complex question preparing methods to terabytes while empowering intelligent reaction times is a noteworthy open exploration issue today.

An issue with current Big Data analysis is the absence of coordination between database frameworks, which have the data and give SQL questioning, with investigation bundles that perform different types of non-SQL preparing, for example, data mining and measurable examinations. Today's investigators are obstructed by a repetitive procedure of sending out data from the database, performing a non-SQL process and bringing the data back. This is a deterrent to continuing the intelligent style of the original of SQL-driven OLAP frameworks into the data mining kind of analysis that is in expanding request. A tight coupling between decisive question dialects and the elements of such bundles will advantage both expressiveness and execution of the analysis.

\subsection{Interpretation of Data}

Being able to investigate Big Data is of constrained worth if clients can't comprehend the analysis. At last, a chief, furnished with the consequence of analysis, needs to decipher these outcomes. This translation can't happen in a vacuum. Generally, it includes looking at all the suppositions made and remembering the analysis. Besides, as we saw above, there are numerous conceivable wellsprings of blunder: PC frameworks can have bugs, models quite often have presumptions, and results can be founded on incorrect data. For these reasons, no mindful client will surrender power to the PC framework. Maybe she will attempt to comprehend, and confirm, the outcomes delivered by the PC. The PC framework must make it simple for her to do as such. This is especially a test with Big Data because of its intricacy. There are regularly essential presumptions behind the data recorded. Systematic pipelines can frequently include various steps, again with presumptions worked in. The late home loan related stun to the money related framework significantly underscored the requirement for such chief industriousness as opposed to acknowledge the expressed dissolvability of a budgetary establishment at face esteem, a leader needs to look at fundamentally the numerous suppositions at different phases of analysis.

To put it plainly, it is once in a while enough to give only the outcomes. Maybe, one must give supplementary data that clarifies how every outcome was determined, and based upon decisively what inputs. Such supplementary data is known as the provenance of the (outcome) data. By concentrate how best to catch, store, and inquiry provenance, in conjunction with methods to catch sufficient metadata, we can make a framework to furnish clients with the capacity both to decipher scientific results acquired and to rehash the analysis with various presumptions, parameters, or data sets.

\section{CHALLENGES IN BIG DATA}

Big data is set to offer organizations enormous understanding. Yet, with terabytes and petabytes of data pouring into associations today, customary structures and foundations are not up to the test. IT groups are troubled with continually developing solicitations for data, impromptu analyses and erratic reports. Chiefs get to be baffled since it takes hours or days to motivate answers to addresses, if by any means. More clients are anticipating that self-administration access should data in a structure they can without much of a stretch comprehend and impart to others. This makes one wonder: How would you display huge data in a way that business pioneers can rapidly comprehend and 
utilize? This is not a minor thought. Mining a large number of columns of data makes a major migraine for examiners tasked with sorting and showing data. Associations regularly approach the issue in one of two ways: Build "samples" with the goal that it is less demanding to both investigate and present the data, or make layout diagrams and charts that can acknowledge certain sorts of data. Both methodologies miss the potential for big data.

\subsection{Security Problems}

These days we share our own data, for example, our preferences, hates and our whereabouts all the more every now and again over the web. These foot shaped impressions might prompt different security issues like area exposure, individual data divulgence.

\subsubsection{Requirement for Security}

Because of perpetual increment in the web utilization the security remains an unsolved puzzle still at this point. These security concerns by and large happen because of absence of education in protection.

\subsubsection{Obstacles for Security}

a. Distinctive Security: At the point when the data around a specific distinct is consolidated together with a vast datasets, there exists a reasonable probability of recognizing the different new concealed bits of knowledge about the specific person which he/she is unconscious[8].

b. Business Security: The different data and the logs, for example, exchange logs, server logs can uncover private data that would give a chance to abuse the data. For instance if organization $\mathrm{A}$ and $\mathrm{B}$ are business contenders then both $\mathrm{A}$ and $\mathrm{B}$ must not uncover any data between one another as this may bring about loss of business security.

c. Under Privileged Security: By investigating the vast datasets it is anything but difficult to recognize the under special ones. The present online networking and different assets make this much simpler. Consequently under advantaged protection can be in question.

\subsection{Inconsistency Problems}

In circumstances where big data are delivered, obtained, totaled, changed, or spoke to, irregularities constantly discover their way into extensive datasets. This can be ascribed to various components in human practices and in choice making process. Once caught in big data, conflicting or clashing marvels can happen at different granularities of learning substance, from data, data, information, metalearning, to ability, and can antagonistically influence the nature of the results in huge data examination process. Irregularities can likewise emerge in thinking strategies, heuristics, or critical thinking methodologies conveyed for different investigation errands, bringing about entanglements for big data examination. At the point when a few vast datasets are consolidated together which has an alternate inception the irregularities will undoubtedly happen. Also this present reality is inclined to have conflicting data. For the most part the irregularity in data happens because of clashing data. In the event that these irregularities are not took care of appropriately then the data sets would not have any uprightness. The inconsistencies can be a noteworthy issue in different fields, for example, heuristics, critical thinking, research examination and business forecast investigation. Keeping in mind the end goal to get a reasonable picture about where the irregularities creep in we should first break down the level of the content in big data.

\subsubsection{Levels of Big Data}

a. Data: The data is the preparatory level in big data. They are essential and less organized type of the content of big data. For the most part the data are content, graphical content, video, sound, images and numeric.

b. Knowledge: They are very much organized and more improved representation among the substance in the data sets. Generally the irregularities are dispensed with at this level however at uncommon situation the irregularities might happen. Consequently mind must be taken while changing over the substance from data to learning.

c. Information: The data is mostly organized thus it is to a degree less demanding to recognize the irregularity. The data more often than not gives an additional quality to the data.

\subsubsection{Types of Inconsistencies:}

Before settling on the most proficient method to run about with the irregularities found in big data, we have to perceive distinctive sorts of irregularities for various sorts of big data. Case in point, for area based data, transient or spatial irregularities will rule, while for unstructured content data, irregularities originating from syntactic, semantic, and down to business circumstances of a characteristic dialect will possess a summoning position. How we distinguish and separate classes of conflicting marvels at levels of data, data, information, Meta learning will prepare for consequent taking care of assignments. Irregularities at data level include different sorts of qualities (typical, numeric, all out, waveform, and so on.). Irregularities at data level show as far as practical conditions or affiliations. At learning level, irregularities show in revelatory or procedural convictions. Meta-information irregularities are shown through control systems or learning choices [9]. These types of inconsistencies are described here.

a. Temporal Inconsistencies: At the point when datasets contain a temporal characteristic, data things with clashing circumstances might correspond or cover in time. The time interim connections between clashing data things can bring about halfway temporal inconsistency or complete temporal inconsistency.

b. Text Inconsistencies: The Text inconsistencies for the most part begin from different sources such as messages, web journals, online networking and so forth. These inconsistencies can very change the uprightness of the information. At whatever point two writings are alluding to the same occasion or entity, then they are said to be co-reference. The co-reference is a required condition for content inconsistencies.

c. Spatial Inconsistencies: The spatial data comprise of geometrical properties and have different spatial relations. The infringement in the spatial imperatives results in the inconsistencies. The spatial inconsistency might happen in reference to spatial relations, for example, topological, directional and distance [10]. 
d. Functional Dependency Inconsistencies: Numerous big datasets are put away, totaled, and cleaned through the assistance of social database frameworks where utilitarian conditions (FD) [12] or restrictive useful conditions [11] assume a basically imperative part in authorizing the trustworthiness requirements for the database.

\subsubsection{Manipulating Inconsistencies:}

As specified in before, the inconsistencies are hard to handle notwithstanding the type of the substance. The datasets must be purged down before it is taken care of in order to dispense with greatest plausibility of inconsistency event. The different simulated visualization tools, machine learning procedures, design investigation ought to be performed to destroy inconsistency in data

\section{NEED OF MODIFIED SYSTEM ARCHITECTURE}

With Big Data, the utilization of discrete frameworks in this style turns out to be restrictively costly given the substantial size of the data sets. The cost is expected not just to the expense of the frameworks themselves, additionally an ideal opportunity to stack the data into various frameworks. In result, Big Data has made it important to run heterogeneous workloads on a solitary framework that is adequately adaptable to handle every one of these workloads. The test here is not to manufacture a framework that is in a perfect world suited for all handling undertakings. Rather, the need is for the hidden framework design to be sufficiently adaptable that the segments based on top of it for communicating the different sorts of preparing errands can tune it to effectively run these diverse workloads. On the off chance that clients are to form and fabricate complex explanatory pipelines over Big Data, it is vital that they have proper abnormal state primitives to determine their requirements in such adaptable frameworks. The MapReduce system has been immensely significant, however is just an initial step. Indeed, even explanatory dialects that endeavor it, for example, Pig Latin, are at a fairly low level with regards to complex examination undertakings. Comparative revelatory particulars are required at larger amounts to meet the programmability and piece needs of these examination pipelines. Other than the fundamental specialized need, there is a solid business basic too. Organizations ordinarily will outsource Big Data preparing, or numerous parts of it. Definitive particulars are required to empower in fact significant administration level agreements, since the purpose of the out-sourcing is to determine unequivocally what undertaking will be performed without going into subtle elements of how to do it.

\section{CONCLUSION}

In this paper we have expressed different security and inconsistency problems that keep on frequenting Big Data examination. The security problems ought to be address by entirely upholding the security laws and by likewise making a safe framework while taking care of client data. So also the inconsistencies must be wiped out as ahead of schedule as could be allowed to enhance data quality there by giving effective data investigation. The difficulties incorporate the conspicuous issues of scale, as well as heterogeneity, absence of structure, blunder taking care of, security, auspiciousness, provenance, and visualization, at all phases of the investigation pipeline from data obtaining to result translation. These specialized difficulties are regular over an expansive assortment of utilization areas, and in this way not practical to address in the connection of one space alone. Besides, these difficulties will require trans-developmental arrangements, and won't be tended to normally by the up and coming era of modern items. The commitment of this work lies in the way that articulating expressly the sorts of conflicting wonders in big data can make ready to enhance the nature of big data investigation.

\section{REFERENCES}

[1] Global Research Data Infrastructures: Towards a 10year vision for global research data infrastructures. Final Roadmap, 2012. [Online].Available:http://www.grdi2020.eu/Repository/ FileScaricati/6bdc07fb-b21d-4b90-81d4d909fdb96b87.pdf

[2] Y.Demchenko, P.Membrey, P.Grosso, C. de Laat, "Addressing Big Data Issues in Scientific Data Infrastructure," in First International Symposium on Big Data and Data Analytics in Collaboration (BDDAC 2013). Part of The 2013 Int. Conf. on Collaboration Technologies and Systems (CTS 2013), May 20-24, 2013, San Diego, California, USA.

[3] Gartner Group, Gartner's 2014 Hype Cycle for Emerging Technologies Maps the Journey to Digital Business,

2014 , http://www.gartner.com/newsroom/id/2819918.

[4] https://en.wikipedia.org/wiki/Big_data

[5] J.Gantz and David Reinsel, Extracting Value from Chaos, IDC IVIEW, June 2011. [Online]. Available: http://www.emc.com/collateral/analyst-reports/idcextracting-value-from-chaos-ar.pdf

[6] The Big Data Long Tail. Blog post by Jason Bloomberg, January 17, 2013. [Online]. Available: http://www.devx.com/blog/the-big-data-long-tail.html

[7] Definting Big Data Architetcure Framework: Outcome of the Brainstorming Session at the University of Amsterdam, 17 July 2013. Presented at NBD-WG, 24 July 2013 [Online]. Available: http://bigdatawg.nist.gov/_uploadfiles/M0055_v1_7606 723276.pdf

[8] Meiko Jenson, "Challenges of Privacy Protection in Big data Analytics", Big Data (Big Data Congress), 2013 IEEE International Congress on IEEE, pp. 235-239.

[9] [9] D. Zhang and E. Gregoire, The landscape of inconsistency: a perspective, International Journal of Semantic Computing, Vol. 5, No.3, 2011, pp.235-256.

[10] A. Rodriguez, Inconsistency issues in spatial databases, in L. Bertossi et al (eds.) Inconsistency Tolerance, LNCS 3300, Springer-Verlag, 2004, pp.237-269.

[11] W. Fan, F. Geerts, X. Jia, and A. Kementsietsidis, Conditional functional dependencies for capturing data inconsistencies, ACM Transactions on DatabaseSystems, Vol.33,Issue2,June2008.

[12] M. V. Martinez, A. Pugliese, G. I. Simari, V. S. Subrahmanian, and H. Prade, How dirty is your relational database? An axiomatic approach, in Proc. 9th European Conference on Symbolic and Quantitative Approaches to Reasoning with Uncertainty, Hammamet, Tunisia, LNAI 4724, 2007, pp.103-114. 